\title{
Aflibercept versus Ranibizumab as a Second Line Therapy After Bevacizumab for Diabetic Macular Edema
}

\author{
Nasser G Alsaedi $\mathbb{D}^{1,2}$ \\ Ruba M Alselaimy ${ }^{3}$ \\ Abdulaziz A Alshamrani (iD) \\ Muhammed AIAjmi ${ }^{4}$ \\ Rajiv Khandekar (D) \\ Hassan Al-Dhibi ${ }^{\text {I }}$ \\ Abdulelah A Al-Abdullah \\ 'King Khaled Eye Specialist Hospital, \\ Riyadh, Saudi Arabia; ${ }^{2}$ King Abdullah \\ Medical City, Makkah, Saudi Arabia; \\ ${ }^{3}$ College of Medicine, King Saud \\ University, Riyadh, Saudi Arabia; ${ }^{4}$ Albahar \\ Eye Centre, Kuwait City, Kuwait
}

Purpose: To compare the visual and anatomic outcomes of aflibercept versus ranibizumab as a second line treatment for persistent diabetic macular edema (DME) after initial bevacizumab injections.

Methods: In this retrospective cohort study, patients with center-involved DME of $\geq 300 \mu \mathrm{m}$ thickness after bevacizumab intravitreal injections in 2015-2019 were included. Those treated with ranibizumab (R) and aflibercept (A) were grouped as group R and group A, respectively. The change in central macular thickness (CMT) measured by optical coherence tomography (OCT) and the best corrected distance visual acuity (BCVA) before and after three-monthly anti-VEGF injections (anti-VEGF) in group $\mathrm{R}$ and group A were compared and reviewed.

Results: There were 80 eyes of 75 patients in group $\mathrm{R}$ and 80 eyes of 72 patients in group A. The initial bevacizumab injections in group $R$ and group A varied significantly $(p=0.01)$. The median change of the CMT after the three injections was not significantly different in group $\mathrm{R}(80 \mu \mathrm{m})$ and group A $(81.5 \mu \mathrm{m})(\mathrm{p}=0.7)$. The improvement of BCVA in group $\mathrm{R}$ and group A was not significant $(\mathrm{p}=0.5)$. Dry macula was noted in 1 vs 14 eyes in group $\mathrm{R}$ vs group $\mathrm{A}$.

Conclusion: After treating refractory DME with initial bevacizumab injections, 3 injections of either aflibercept or ranibizumab had similar anatomic and functional outcomes. Aflibercept achieved dry macula in more eyes with refractory DME compared to ranibizumab.

Keywords: aflibercept, bevacizumab, persistent DME, ranibizumab, VEGF switch, vascular endothelial growth factor

\section{Introduction}

Intra-vitreous (IV) injections of anti-VEGF agents is the current mode of DME management. ${ }^{1}$ However refractory DME after IV anti-VEGF is a challenge to the retina specialists. Few studies provide insight into the options of treatment in cases of poor response to the initial therapy. ${ }^{4}$ Switching to another anti-VEGF can provide additional efficacy in refractory DME. ${ }^{2}$

The efficacy of different anti-VEGF for the initial treatment of DME with direct head to head comparison was reported previously. ${ }^{1}$ Switching to aflibercept was reported by Lim et al after initial therapy of ranibizumab and bevacizumab. ${ }^{5}$ The financial burden of different treatment regimens with anti-VEGF need to be considered whenever the decision of treatment is made. ${ }^{6}$ 
To the best of our knowledge, outcome comparisons of aflibercept and ranibizumab as a second line of treatment for refractory DME after initial bevacizumab injections have not been studied.

We present anatomic and functional outcomes of aflibercept versus ranibizumab as a second line treatment for diabetic macular edema in a tertiary hospital in Saudi Arabia.

\section{Materials and Methods}

In this is retrospective study, patients with refractory DME after initial therapy with bevacizumab injections were treated with one of the two anti-VEGF agents between March 2015 and July 2019 at a tertiary eye hospital in central Saudi Arabia. After at least three injection of bevacizumab the medication was switched to other antiVEGF for addressing the residual DME. Our study was approved by the institutional review board (1905-R) at King Khaled Eye Specialist Hospital (KKESH) and was conducted in accordance with the tenets of the Declaration of Helsinki for research involving humans. Patient consent to review their medical records was not required by the Institutional Review Board of KKESH because no identifiable data were obtained and all patients' data was kept confidential. Exclusion criteria included pregnant women, patients with uveitis and patients with tractional type of DME.

To calculate the sample size, we assumed, based on previous studies, that the reduction in CST in ranibizumab group would be $(265.4 \mu)$ in SC-OCT and in aflibercept group the reduction in CST would be $(191.1 \mu) .{ }^{7,8}$ We used open Epi software to calculate the sample size to get $95 \mathrm{CI}$ and $90 \%$ power to the study with the ratio $1: 1$ in two arms. ${ }^{9}$ We required 71 eyes in each arm, $71: 71$, but to address the possible loss of data and missed clinical follow up, we increased the sample size to 80:80 eyes in each arm.

All patients having center-involved DME, defined as a central subfield thickness (CST) of more than 300 microns on spectral-domain optical coherence tomography (SD-OCT; Spectralis; Heidelberg Engineering, Heidelberg, Germany), after completion of initial bevacizumab injections of three to six doses with suboptimal response followed by a shift to either ranibizumab injection $(0.5 \mathrm{mg} /$ $0.05 \mathrm{~mL})$ or aflibercept injection $(2 \mathrm{mg} / 0.05 \mathrm{~mL})$ were included.

In both ranibizumab (group R) and aflibercept group (group A), the first evaluation after completion of bevacizumab initial doses was considered as the baseline for the visual acuity and central macular thickness measurement, which was 4 weeks \pm 1 week from the last dose of bevacizumab.

The primary successful outcome was defined as the change in central macular thickness from baseline compared to 4 weeks ( \pm 1 week) measurement after completion of the three aflibercept or ranibizumab injections. A partial response was defined as a reduction of more than $10 \%$ in CST, with residual edema (CST is more than $300 \mathrm{~mm}$ or with the presence of cystic edema on SDOCT). A non-responder was defined as less than $10 \%$ reduction in CST at 4-5 weeks following the last injection compared to the baseline. The secondary successful outcome was a two-line improvement in BCVA (best corrected visual acuity) at last follow up compared to that at baseline. The visual impairment grades were 'normal functional vision (best corrected vision 20/20 to 20/60, moderate visual impairment (BCVA between 20/60 and 20/ 200), severe visual impairment (BCVA $<20 / 200$ to $20 /$ $400)$ and blind (BCVA <20/400). ${ }^{10}$

The data were collected on a pretested data collection form and entered into an Excel ${ }^{\circledR}$ spreadsheet (Microsoft Corp., Redmond, WA, USA). Demographic information was analyzed per person and outcomes were reviewed per eye as a unit. Statistical Package for Social Studies (SPSS 25) (IBM Corp., Armonk, NY, USA) was used for univariate analysis. The qualitative variables were presented using frequencies and percentage proportions. The quantitative variables were evaluated for a normal distribution. If they were normally distributed, we presented the mean and standard deviations. If the quantitative variables were not normally distributed, then we presented their median and interquartile ranges. To compare the outcomes in two groups, we calculated relative risk, $95 \%$ confidence interval (CI) and two-sided p-value. A p-value of $<0.05$ was considered as statistically significant. In case of skewed data, a nonparametric method was used to compare subgroups. The Mann-Whitney $U$-test p-value was calculated if two subgroup variables were compared. For comparison of more than 2 subgroups, we calculated the Kruskal-Wallis p-value.

\section{Results}

There were 80 eyes of 75 patients in ranibizumab group and 80 eyes of 72 patients in aflibercept group. The mean age of the patients was 63.4 years in ranibizumab group and 62.5 in aflibercept group; $p=0.4$. All patients in both groups had 
type two diabetes. The mean duration of diabetes was 17.6 years in ranibizumab group and 16 years in aflibercept group $(\mathrm{p}=0.8)$. At baseline in ranibizumab group, proliferative diabetic retinopathy (PDR) was found in 43.2\% (35) eyes while in the aflibercept group PDR was found in $26.3 \%$ (21) eyes $(p=0.05)$. Non-proliferative diabetic retinopathy stages in ranibizumab group were mild in $9.9 \%$, moderate in $29.6 \%$ and severe in $17.3 \%$ while in aflibercept group there were $16.3 \%$ mild, $35 \%$ moderate and $22.5 \%$ severe NPDR ( $p=$ 0.04). Panretinal photocoagulation treatment prior to the course of ranibizumab or aflibercept, was noticed to be $35.8 \%$ versus $23.8 \%$, respectively $(p=0.04)$. Two-thirds of the patients in both groups were phakic at baseline. Previous vitrectomy was observed in two patients with silicone oil in ranibizumab group.

The median number of initial bevacizumab injections was 3 injections in ranibizumab group compared to 6 injections in aflibercept group $(p=0.01)$.

The central macular thickness CMT at baseline (after initial bevacizumab injections) was significantly higher in ranibizumab group compared to aflibercept group; 514.5 $\pm 148.6 \mu$ versus $453 \pm 157 \mu$, respectively $(p=0.006)$.

The visual acuity grades in group $\mathrm{R}$ and group A were not statistically significant $(\mathrm{p}=0.9$ ).

All eyes in both groups had received three injections of the second line (ranibizumab or aflibercept) every month except two eyes in both groups which had received more than three injections.

There was significant reduction in the CMT after both type of injections compared to baseline $(p<0.001)$. The central macular thickness before and after the 3 injections of anti-VEGF of two types is given in Table 1.
The mean difference in the CMT was not statistically different between ranibizumab vs aflibercept injections (90 microns vs 99 microns, respectively) $(\mathrm{p}=0.7)$.

In term of visual acuity there was no significant difference between both groups after therapy; $\mathrm{p}$-value $=0.9$ (Table 2).

In ranibizumab group, $23.5 \%$ eyes had improved vision by 'two or more lines' compared to $20 \%$ of the eyes in aflibercept group. Forty (50\%) eyes in both groups had unchanged BCVA. One-third of the patients in both groups showed decrease in the BCVA compared to the baseline (27.2\% in ranibizumab group versus $30 \%$ in aflibercept group). The improvement in BCVA of 'two or more lines' was similar in both groups $(\mathrm{p}=0.5)$.

The reduction of CMT was correlated to different pre-shift variables and it was noted that regression analysis suggested that reduction of CMT after 3 injections of anti-VEGF could be predicted by knowing type of intervention as second line of treatment (ranibizumab vs aflibercept) and CMT before start of 2nd line of treatment. The number of previous bevacizumab injections $(\mathrm{p}=0.13)$ and the status of diabetic retinopathy $(\mathrm{p}=$ 0.3 ) were not significantly influencing the correlation of CMT reduction to the type of intervention as second line of treatment Table 3.

In aflibercept group, in $19(23.8 \%)$ eyes clinicians discontinued further anti-VEGF treatment after completing the three injections; 14 out of those 19 eyes had resolved DME and required no further injection at one month post the three injections of aflibercept. While 2 of 19 patients shifted to focal laser treatment after the CMT improvement, another three patients refused further injections. Only one eye stopped injection in ranibizumab group due to lost follow up.

Table I Central Macular Thickness in Eyes with Refractory Diabetic Macular Edema Before and After Three Injections of AntiVascular Growth Factors as Second Line of Treatment After 3 to 6 Intravitreal Injections of Bevacizumab

\begin{tabular}{|c|c|c|c|c|c|}
\hline & \multicolumn{2}{|c|}{ Ranibizumab Treatment Regimen } & \multicolumn{2}{|c|}{ Aflibercept Treatment Regimen } & \multirow{2}{*}{$\begin{array}{r}\text { Mean Difference }(95 \% \mathrm{Cl}) \\
\text { p-value }\end{array}$} \\
\hline & Mean $(\mu)$ & $\operatorname{SDV}(\mu)$ & Mean $(\mu)$ & SDV $(\mu)$ & \\
\hline Before treatment & $5 \mid 4.5$ & 148.6 & 453.2 & 127.2 & $\begin{array}{r}61.3 \\
(18.2 ; 104.3) \\
P=0.006\end{array}$ \\
\hline After treatment regimen & 424.3 & 129.1 & 353.9 & 112.5 & $\begin{array}{r}70.4 \\
(32.8 ; 108.8) \\
P<0.001\end{array}$ \\
\hline $\begin{array}{l}\text { Matched pair analysis: } \\
\text { Mean difference } \\
(95 \% \mathrm{Cl}) \mathrm{P} \text { value }\end{array}$ & & $\begin{array}{r}90.6 \\
(62.5 ; 117.8) \\
P<0.001\end{array}$ & & $\begin{array}{r}99.3 \\
(74.7 ; 123.9) \\
P<0.001\end{array}$ & \\
\hline
\end{tabular}


Table 2 Visual Impairment Grades in Eyes with Refractory Diabetic Macular Edema Before and After Three Injections of Anti-Vascular Growth Factors as Second Line of Treatment After 3 to 6 Intravitreal Injections of Bevacizumab

\begin{tabular}{|c|c|c|c|c|c|c|}
\hline & & \multicolumn{2}{|c|}{$\begin{array}{c}\text { Ranibizumab Treatment } \\
\text { Regimen }\end{array}$} & \multicolumn{2}{|c|}{$\begin{array}{c}\text { Aflibercept Treatment } \\
\text { Regimen }\end{array}$} & \multirow[t]{2}{*}{ Validation } \\
\hline & & Number & Percentage & Number & Percentage & \\
\hline \multirow[t]{4}{*}{ Before treatment } & $20 / 20$ to $20 / 60$ & 37 & 45.7 & 32 & 40.0 & $\chi^{2}=0.4 \mathrm{df}=4$ \\
\hline & $<20 / 60$ to $20 / 200$ & 32 & 39.5 & 43 & 53.8 & $p=0.7$ \\
\hline & $<20 / 200$ to $20 / 400$ & 10 & 12.3 & 5 & 6.3 & \\
\hline & $<20 / 400$ & 2 & 2.5 & 0 & 0.0 & \\
\hline \multirow[t]{4}{*}{ After treatment regimen } & $20 / 20$ to $20 / 60$ & 36 & 44.4 & 36 & 45 & $\chi^{2}=0.02 \mathrm{df}=4$ \\
\hline & $<20 / 60$ to $20 / 200$ & 36 & 44.4 & 36 & 45 & $p=0.9$ \\
\hline & $<20 / 200$ to $20 / 400$ & 8 & 9.9 & 8 & 10 & \\
\hline & $<20 / 400$ & 1 & 1.2 & 0 & 0 & \\
\hline Validation & & \multicolumn{2}{|r|}{$\begin{array}{r}\chi^{2}=0.05 \mathrm{df}=4 \\
P=0.8\end{array}$} & \multicolumn{2}{|r|}{$\begin{aligned} \chi^{2}=0 \mathrm{df} & =4 \\
\mathrm{P} & =1\end{aligned}$} & \\
\hline
\end{tabular}

Table 3 Predictors of Reduction of Central Macular Thickness Following Anti-Vascular Growth Factor Treatment Regimen in Eyes with Refractory Diabetic Macular Edema After 3 to 6 Intravitreal Injections of Bevacizumab

\begin{tabular}{|c|c|c|c|c|}
\hline & $\begin{array}{r}\text { Unstandardized } \\
\text { Coefficients }\end{array}$ & $\begin{array}{r}\text { Standardized } \\
\text { Coefficients (B) }\end{array}$ & $\begin{array}{r}\text { 95\% Confidence } \\
\text { Interval of B }\end{array}$ & $\begin{array}{r}\text { Two-sided } \\
\text { p-value }\end{array}$ \\
\hline Constant & 203.7 & & $49.1 ; 358.2$ & 0.010 \\
\hline Type of anti-VEGF treatment regimen & -47.2 & -0.196 & $-94.5 ; 0.1$ & 0.050 \\
\hline CMT before treatment regimen & -0.5 & -0.538 & $-0.6 ;-0.3$ & 0.000 \\
\hline Grade of diabetic retinopathy & 12.6 & 0.108 & $-10.0 ; 36.0$ & 0.290 \\
\hline Duration of diabetes & -3.0 & -0.167 & $-6.5 ; 0.5$ & 0.093 \\
\hline Number of bevacizumab injection in past & 11.2 & 0.149 & $-3.3 ; 25.8$ & 0.130 \\
\hline
\end{tabular}

After the three injections of the second line therapy, 36 (44\%) eyes in ranibizumab group continued on ranibizumab and $44(54.3 \%)$ eyes were shifted to aflibercept. In aflibercept group, majority of eyes continued on aflibercept, 56 $(70 \%)$ eyes, and only $5(6.3 \%)$ eyes were switched to ranibizumab following the three consecutive aflibercept injections. Continuation of aflibercept after completed second line treatment was significantly more compared to ranibizumab, and the chance for shifting to another anti-VEGF were significantly more after ranibizumab treatment $(\mathrm{p}<0.001)$. No ocular complications (endophthalmitis or elevated intraocular pressure) were observed among both groups.

\section{Discussion}

Widely-used and cost-effective bevacizumab injection regimens have shown that as high as $40-65.6 \%$ of eyes have residual DME. ${ }^{11,12}$ Hence switching to alternative anti-VEGF agents is crucial and has shown promising anatomic and functional outcomes. ${ }^{5,12,13}$ However, which anti-VEGF to select is still debatable and present study could not get sufficient evidence to recommend aflibercept or ranibizumab treatment regimen as 2 nd line of treatment.

In the present study, we noted that a three-injection regimen of both anti-VEGF ranibizumab and aflibercept in eyes with refractory DME after treatment of intra-vitreal bevacizumab had a similar effect on the reduction of CMT and no significant difference in the visual gain. However, more eyes seem to achieve dry macula after aflibercept than ranibizumab treatment regimen. In addition, more patients had been switched to or kept on aflibercept after the three injections of the second line therapy in both groups (aflibercept and ranibizumab group) to address the residual DME.

To the best of our knowledge, our study is unique in comparing two commonly used anti-VEGF in refractory DME after 3 to 6 bevacizumab injection regimen. Demircan et $\mathrm{al}^{12}$ studied the effect of continuing on ranibizumab vs switching to aflibercept injection after initial three injections of ranibizumab. In contrast to our study findings, they noted better outcomes of aflibercept treatment regimen than ranibizumab treatment regimen in term of the reduction 
in mean CMT. In another study but for treating macular edema due to age-related macular degeneration, Waizel et $\mathrm{al}^{8}$ reported superiority of aflibercept treatment regimen over ranibizumab and bevacizumab treatment.

In view of higher binding affinity to VEGF-A, aflibercept binds better to VEGF-B and placental growth factor (P1GF) compared to bevacizumab or ranibizumab and therefore has better bioavailability at the macula. ${ }^{14}$ This logic agrees with better anatomic outcomes reported following switch to aflibercept treatment regimen but could not explain no visual improvement found in a study by Rihamy et al. ${ }^{15}$ Wood et $\mathrm{al}^{7}$ also demonstrated superiority of aflibercept treatment regimen in eyes with refractory DME after multiple initial bevacizumab or ranibizumab injections.

Reduction of macular thickness noted in our study did not match with vision improvement following treatment of refractory DME. The anti-VEGF regimen could cause physio-pathological alteration in macular thickness and resorption of interstitial fluid and thus OCT-based retinal thickness could be brought to near normal. However, retinal cells being nerve cells and not having regenerating power like other brain cells, damage to rods and cones in macular region due to DME is less likely to be reverted.

The cases allotted to ranibizumab and aflibercept groups in present study were influenced by the practice pattern in our institute where bevacizumab used to be given for three injections before physicians can switch to ranibizumab. More recently, patients will be switched to other anti-VEGF only after 6 initial injections of bevacizumab. Hence the cases in aflibercept group had significantly more number of prior bevacizumab injections. Despite that, in multivariate regression analysis the number of prior bevacizumab injections did not influence the outcome ie reduction in CMT.

In our study, after completing the second line of 3 consecutive anti-VEGF injections, eyes that continued on aflibercept treatment were more than those continued with ranibizumab. The clinical decision to shift to newly available aflibercept treatment could be due to perceived benefits over ranibizumab.

There were limitations in this study. This being a retrospective cohort, random allocation was not possible. It could therefore have been influenced by selection bias. Of note is that CMT was lower in aflibercept group to start with; however, this was overcome by evaluating the reduction in the CMT compared to baseline rather than the final CMT in both groups.
In addition, selection of two comparison groups of different time-frames and having different number of initial bevacizumab injections could be a reason for our non-conclusive study outcomes.

The information on glycemic control including HbA1c level, amount of retinal ischemia, fluorescein angiography study and other comorbidities, eg systemic hypertension or renal failure etc, was not collected. ${ }^{14}$ Thus differential impact of anti-VEGF treatment regimen could be influenced by differential primary prevention status of diabetic retinopathy. Study with better study design like prospective cohort or randomized clinical trial will confirm our study outcomes.

Evidence from the present study is still inconclusive for recommending a shift from initial bevacizumab to aflibercept or ranibizumab. Availability, cost of the treatment regimen and introduction of newer modalities of steroid implants or laser should be taken into account before clinicians decide about selection of second line therapy for refractory DME.

\section{Acknowledgments}

Mrs. Muneera Alfutais who assisted in the data entry.

\section{Disclosure}

The authors declare that they have no conflicts of interest.

\section{References}

1. Diabetic Retinopathy Clinical Research Network. Aflibercept, bevacizumab, or ranibizumab for diabetic macular edema. $N$ Engl $J$ Med. 2015;372(13):1193-1203. doi:10.1056/NEJMoa1414264

2. Hussain RM, Ciulla TA. Treatment strategies for refractory diabetic macular edema: switching anti-VEGF treatments, adopting corticosteroid-based treatments, and combination therapy. Expert Opin Biol Ther. 2016;6(3):365-374. doi:10.1517/14712598.2016. 1131265

3. Busch C, Zur D, Fraser-Bell S, et al. Shall we stay, or shall we switch? Continued anti-VEGF therapy versus early switch to dexamethasone implant in refractory diabetic macular edema. Acta Diabetol. 2018;55 (8):789-796. doi:10.1007/s00592-018-1151-x

4. Lim LS, Ng WY, Mathur R, et al. Conversion to aflibercept for diabetic macular edema unresponsive to ranibizumab or bevacizumab. Clin Ophthalmol. 2015;9:1715-1718. doi:10.2147/ OPTH.S81523

5. Wood EH, Karth PA, Moshfeghi DM, Leng T. Short-term outcomes of aflibercept therapy for diabetic macular edema in patients with incomplete response to ranibizumab and/or bevacizumab. Ophthalmic Surg Lasers Imaging Retina. 2015;46(9):950-954. doi:10.3928/2325816020151008-08

6. Waizel M, Todorova MG, Masyk M, et al. Switch to aflibercept or ranibizumab after initial treatment with bevacizumab in eyes with neovascular AMD. BMC Ophthalmol. 2017;17(1):79. doi:10.1186/ s12886-017-0471-x 
7. Dean AG, Sullivan KM, Soe MM OpenEpi: open source epidemiologic statistics for public health, version. 2013. Available from: www. OpenEpi.com. Accessed October 1, 2018.

8. Dandona L, Dandona R. Revision of visual impairment definitions in the international statistical classification of diseases. BMC Med. 2006;4(1):7. doi:10.1186/1741-7015-4-7

9. Bressler NM, Beaulieu WT, Glassman AR, et al. Persistent macular thickening following intravitreous aflibercept, bevacizumab, or ranibizumab for central-involved diabetic macular edema with vision impairment: a secondary analysis of a randomized clinical trial. JAMA Ophthalmol. 2018;136(3):257-269. doi:10.1001/jamaophthalmol.2017. 6565

10. Bressler SB, Ayala AR, Bressler NM, et al. Persistent macular thickening after ranibizumab treatment for diabetic macular edema with vision impairment. JAMA Ophthalmol. 2016;134(3):278-285. doi:10. 1001/jamaophthalmol.2015.5346

11. Klein KA, Cleary TS, Reichel E. Effect of intravitreal aflibercept on recalcitrant diabetic macular edema. Int J Retin Vitr. 2017;3(1):16. doi:10.1186/s40942-017-0064-0
12. Demircan A, Alkin Z, Yesilkaya C, et al. Comparison of intravitreal aflibercept and ranibizumab following initial treatment with ranibizumab in persistent diabetic macular edema. $J$ Ophthalmol. 2018;2018. doi:10.1155/2018/4171628

13. Brown DM, Schmidt-Erfurth U, Do DV, et al. Intravitreal aflibercept for diabetic macular edema: 100-week results from the VISTA and VIVID studies. Ophthalmology. 2015;122(10):2044-2052. doi:10. 1016/j.ophtha.2015.06.017

14. Sabanayagam $\mathrm{C}$, Banu $\mathrm{R}$, Chee $\mathrm{ML}$, et al. Incidence and progression of diabetic retinopathy: a systematic review. Lancet Diabetes Endocrinol. 2019;7(2):140-149. doi:10.1016/S2213-8587(18)30 128-1

15. Rahimy E, Shahlaee A, Khan MA, et al. Conversion to aflibercept after prior anti-VEGF therapy for persistent diabetic macular edema. Am J Ophthalmol. 2016;164:118-127. doi:10.1016/j.ajo.2015.12.030
Clinical Ophthalmology

\section{Publish your work in this journal}

Clinical Ophthalmology is an international, peer-reviewed journal covering all subspecialties within ophthalmology. Key topics include: Optometry; Visual science; Pharmacology and drug therapy in eye diseases; Basic Sciences; Primary and Secondary eye care; Patient Safety and Quality of Care Improvements. This journal is indexed on PubMed

\section{Dovepress}

Central and CAS, and is the official journal of The Society of Clinical Ophthalmology (SCO). The manuscript management system is completely online and includes a very quick and fair peer-review system, which is all easy to use. Visit http://www.dovepress.com/ testimonials.php to read real quotes from published authors. 